\title{
Dynamics and phylogenetic relationships of HIV-1 transmitted drug resistance according to subtype in Italy over the years 2000-14
}

\author{
L. Fabeni ${ }^{1}$ t, C. Alteri ${ }^{2 *}$ t, D. Di Carlo ${ }^{2}$, N. Orchi ${ }^{1}$, L. Carioti ${ }^{2}$, A. Bertoli ${ }^{2}$, C. Gori ${ }^{1}$, F. Forbici ${ }^{1}$, F. Continenza ${ }^{1}$, \\ G. Maffongelli ${ }^{3}$, C. Pinnetti ${ }^{1}$, A. Vergori ${ }^{1}$, A. Mondi ${ }^{1}$, A. Ammassari ${ }^{1}$, V. Borghi ${ }^{4}$, M. Giuliani ${ }^{5}$, G. De Carli ${ }^{1}$, S. Pittalis ${ }^{1}$, \\ S. Grisetti ${ }^{1}$, A. Pennica ${ }^{6}$, C. M. Mastroianni ${ }^{7}$, F. Montella ${ }^{8}$, A. Cristaudo ${ }^{5}$, C. Mussini ${ }^{4}$, E. Girardi ${ }^{1}$, M. Andreoni $^{3}$, \\ A. Antinori ${ }^{1}$, F. Ceccherini-Silberstein ${ }^{2}$, C. F. Perno ${ }^{1}$ and M. M. Santoro ${ }^{2}$ on behalf of the SENDIH Study Group \\ ${ }^{1}$ National Institute for Infectious Diseases L Spallanzani, IRCCS, Rome, Italy; ${ }^{2}$ University of Rome Tor Vergata, Rome, Italy; ${ }^{3}$ University \\ Hospital Tor Vergata, Rome, Italy; ${ }^{4}$ Modena University Hospital, Modena, Italy; ${ }^{5}$ San Gallicano Dermatological Institute, IRCCS, Rome, \\ Italy; ${ }^{6}$ S. Andrea Hospital, Rome, Italy; ${ }^{7}$ Sapienza University, Latina, Italy; ${ }^{8}$ S. Giovanni Addolorata Hospital, Rome, Italy
*Corresponding author. Tel: +39-0672596566/6564; Fax: +39-0672596039; E-mail: claudia.alteri@uniroma2.it †These authors made an equal contribution. $\ddagger$ Members are listed in the Acknowledgements.

Received 10 March 2017; returned 24 April 2017; revised 30 May 2017; accepted 9 June 2017

Background: Transmitted drug-resistance (TDR) remains a critical aspect for the management of HIV-1-infected individuals. Thus, studying the dynamics of TDR is crucial to optimize HIV care.

\begin{abstract}
Methods: In total, 4323 HIV-1 protease/reverse-transcriptase sequences from drug-naive individuals diagnosed in north and central Italy between 2000 and 2014 were analysed. TDR was evaluated over time. Maximum-likelihood and Bayesian phylogenetic trees with bootstrap and Bayesian-probability supports defined transmission clusters.

Results: Most individuals were males (80.2\%) and Italian (72.1\%), with a median (IQR) age of 37 (30-45) years. MSM accounted for $42.2 \%$ of cases, followed by heterosexuals (36.4\%). Non-B subtype infections accounted for $30.8 \%$ of the overall population and increased over time $(<2005-14: 19.5 \%-38.5 \%, P<0.0001)$, particularly among Italians $(<2005-14: 6.5 \%-28.8 \%, P<0.0001)$. TDR prevalence was $8.8 \%$ and increased over time in nonB subtypes $(<2005-14: 2 \%-7.1 \%, P=0.018)$. Overall, 467 transmission clusters (involving 1207 individuals; $27.9 \%)$ were identified. The prevalence of individuals grouping in transmission clusters increased over time in both $B(<2005-14: 12.9 \%-33.5 \%, P=0.001)$ and non-B subtypes $(<2005-14: 18.4 \%-41.9 \%, P=0.006)$. TDR transmission clusters were $13.3 \%$ within the overall cluster observed and dramatically increased in recent years $(<2005-14: 14.3 \%-35.5 \%, P=0.005)$. This recent increase was mainly due to non-B subtype-infected individuals, who were also more frequently involved in large transmission clusters than those infected with a B subtype [median number of individuals in transmission clusters: 7 (IQR 6-19) versus 4 (3-4), $P=0.047$ ].
\end{abstract}

Conclusions: The epidemiology of HIV transmission changed greatly over time; the increasing number of transmission clusters (sometimes with drug resistance) shows that detection and proper treatment of the multitransmitters is a major target for controlling HIV spread.

\section{Introduction}

HIV-1 transmitted drug resistance (TDR) is a clinical and public health issue due to its role in compromising the response to ART at the individual and population level, ${ }^{1-4}$ limiting the antiretroviral regimen options. As a result, European and US guidelines recommend genotypic resistance testing in HIV-infected individuals at entry into care (regardless of whether ART will be initiated immediately). ${ }^{5,6}$

To date, estimates of TDR rates vary across the world and range from $3.4 \%$ to $25.2 \%$ in the USA, ${ }^{7-9}$ and from $7.5 \%$ to $11.7 \%$ in
Europe. ${ }^{10-13}$ Despite the general decreasing trend of TDR in both the United States and Europe in the last few years, an alarming increase has been noticed in some specific settings. ${ }^{7,14}$ In this regard, in several countries different frequencies of TDR, according to subtypes and risk factors, have been observed, especially in the last decade, when an increase in non-B subtypes and circulating recombinant forms (CRFs) has been reported. ${ }^{15-17}$ This increase in non-B subtypes occurred in conjunction with associated epidemiological changes such as waves of migration from low-income to middle-income areas, ${ }^{18,19}$ and the increase of HIV transmission

(c) The Author 2017. Published by Oxford University Press on behalf of the British Society for Antimicrobial Chemotherapy. All rights reserved. For Permissions, please email: journals.permissions@oup.com. 
rate among $\mathrm{MSM}^{20,21}$ which to date accounts for at least $43 \%$ of new HIV-1 cases. $^{22,23}$

In this scenario, studying the linkages between viruses through transmission cluster (TC) analysis can be used to provide an in-depth understanding of TDR dynamics and resistance spread. ${ }^{11,24,25}$ For these reasons, this study aims to characterize the dynamics and phylogenetic relationships of TDR and subtypes among a large cohort of HIV-1-infected individuals diagnosed in Italy from 2000 to 2014.

\section{Methods}

\section{Study population}

Between 2000 and 2014, plasma samples from 4323 newly diagnosed HIV-1-infected individuals, naive to ART, attending three different counselling and testing centres in north and central Italy, were tested for antiretroviral drug resistance (DR) genotyping, according to routine clinical practice.

Individuals were defined as recently infected by: (i) clinical/laboratory signs of primary HIV infection (HIV-1 RNA levels $>10000$ copies $/ \mathrm{mL}$ and negative or indeterminate HIV-1 antibody test); (ii) a documented negative HIV-1 test performed within 6 months before the HIV-1 diagnosis; and (iii) an antibody avidity index $\leq 0.80$ (test performed only in clinically AIDSfree individuals). ${ }^{26,27}$

All clinical and virological information used in this study was collected within 8 weeks after the initial HIV-1 diagnosis (range of weeks after HIV-1 diagnosis: 0-8).

\section{Ethics}

This study was approved by the ethics committee of Tor Vergata Hospital (Ethics Approval No. 238/16, 14 December 2016) and L. Spallanzani National Institute for Infectious Diseases, IRCCS (Ethics Approval No. 51, 18 December 2003). The research was conducted on anonymous samples in accordance with the principles of the Declaration of Helsinki and the Italian Ministry of Health. All information, including virological and clinical data, was recorded in an anonymized database.

\section{HIV-1 genotyping}

For all individuals HIV-1 pol [containing the full-length protease (PR) and the first 335 reverse transcriptase (RT) codons] sequences were available at the time of diagnosis (range 0-2 months from diagnosis). HIV-1 pol genotype analyses were performed on plasma samples, as previously described. ${ }^{28}$ All samples were processed immediately upon arrival in clinical laboratories.

\section{HIV-1 subtyping}

For each HIV-1-infected individual, the HIV-1 subtype was determined. pol sequences were aligned and compared with reference sequences for the major HIV-1 subtypes, available at: https://www.hiv.lanl.gov/content/se quence/NEWALIGN/align.html\#ref, using CLUSTAL X. The sequences were then manually edited with the Bioedit program, and gaps were removed from the final alignment. Subtype or CRF assignments were achieved by constructing phylogenetic trees using the neighbour-joining ( $\mathrm{NJ}$ ) method. ${ }^{29}$ Distances were calculated using MEGA 6 based on the Kimura two-parameter model. ${ }^{30}$ The reliability of the branching orders was assessed by bootstrap analysis of 1000 replicates.

\section{Definition of TDR}

TDR was evaluated by considering the WHO-2009 list $^{31}$ with the additional RT mutations K65E/N, E138G/K/Q/R, V179L, G190Q, T215N, H221Y, F227C and M230I, reported in the International AIDS Society (IAS) list ${ }^{32}$ and/or the Stanford 2017 database (https://hivdb.stanford.edu/, last updated 7 February 2017).

HIV-1 strains were defined as resistant if carrying at least one TDR mutation.

\section{Transmission cluster analysis}

TCs, including pairs ( 2 individuals) and clusters ( $\geq 3$ individuals) were first deduced by the NJ method by using all the 4323 pol sequences obtained via routine clinical practice in the period 2000-14. To avoid any possibility of cross-contamination, identical sequences amplified in the same run were excluded. To avoid the influence of convergent evolution at antiretroviral DR mutations, sequences were stripped at positions related to DR. Only clusters with a bootstrap value $>90 \%$ and an average genetic distance $<0.015$ were selected. ${ }^{33}$

The robustness of the TCs was further tested using the maximum likelihood (ML) method and a Bayesian analysis.

The ML tree was inferred with the general time-reversible nucleotide substitution model (GTR) with gamma-distribution among site rate heterogeneity, a proportion of invariable sites $\left(\mathrm{GTR}+\mathrm{I}+\Gamma_{5}\right)^{34}$ and 1000 bootstrap replicates (using the RaxML software, available at http://sco.h-its.org/exe lixis/web/software/raxml/index.html). TCs were recognized by Cluster Picker, ${ }^{35}$ retrieved at http://hiv.bio.ed.ac.uk/software.html, based on a bootstrap value $>90 \%$ and an average genetic distance $<0.015$. The tree was rooted using a midpoint rooting by FigTree software version 1.4.2.

The Bayesian phylogenetic tree was reconstructed with MrBayes, ${ }^{36}$ using a GTR $+\mathrm{I}+\Gamma_{5}$. A Monte Carlo Markov chain search was run for $5 \times 10^{6}$ generations with the trees sampled every 100th generation (with a burn-in of $50 \%) .{ }^{37}$ Statistical support was obtained by calculating the posterior probability of each monophyletic clade, and an a posteriori consensus tree was generated after 50\% burn-in. Clades were considered epidemiological clusters only if a posterior probability of 1 was inferred.

\section{Statistical analysis}

To evaluate potential differences in the prevalence of TDR according to subtype, the $\chi^{2}$ test or Fisher's exact test was used, as appropriate. Potential differences in the prevalence of TDR, subtypes and risk factor over the years 2000-14 were evaluated by time-series analysis for linear trend. The 251 individuals diagnosed between 2000 and 2004 were gathered together in a single time period defined as $<2005$, in order to have a similar numerical distribution of individuals over the years.

Factors associated with TDR (in both the overall population and within clusters) were evaluated by uni-multivariable logistic regression analysis, using as confounders gender, age, subtype, risk factor, nationality, year of diagnosis and sequencing, viraemia and CD4 count at sequencing, type of infection and to be part of TC.

For all statistical tests, the level of significance for the evaluation of two-sided $P$ values was set at $\alpha=0.05$. All the analyses were performed by using the R open source statistical environment (version 3.3.1) and SPSS (version 23) for Windows (SPSS Inc., Chicago, Illinois).

\section{Results}

\section{Patient characteristics}

A total of 4323 individuals newly diagnosed with HIV-1 from 2000 through 2014 were included (Table 1). The majority of these individuals (3468, $80.2 \%$ ) were male, Italian (3115, $72.1 \%$ ) and MSM $(1824,42.2 \%)$. Recent infections accounted for the $22.5 \%$ of the individuals for whom state of infection was available.

The majority ( $N=2992,69.2 \%$ ) of the individuals were infected with a B subtype, followed by CRF02_AG (329, 7.6\%), F1 (248, 5.7\%), 
Table 1. Patient characteristics

\begin{tabular}{|c|c|c|c|c|}
\hline Characteristic & Overall $(N=4323)$ & $B(N=2992)$ & Non-B $(N=1331)$ & $P$ value $^{a}$ \\
\hline Male, $n(\%)$ & $3468(80.2)$ & $2548(85.2)$ & $920(69.1)$ & $<0.001$ \\
\hline \multicolumn{5}{|l|}{ Geographic area, n (\%) } \\
\hline Italy & $3115(72.1)$ & $2492(83.3)$ & $623(46.8)$ & $<0.001$ \\
\hline Africa & $446(10.3)$ & $45(1.5)$ & $401(30.1)$ & $<0.001$ \\
\hline Europe & $355(8.2)$ & $182(6.1)$ & $173(13.0)$ & $<0.001$ \\
\hline America & $328(7.6)$ & $240(8.0)$ & $88(6.6)$ & 0.106 \\
\hline Asia/Australia/Oceania & 79 (1.8) & $33(1.1)$ & $46(3.5)$ & $<0.001$ \\
\hline \multicolumn{5}{|l|}{ Risk factor, $n(\%)$} \\
\hline MSM & $1824(42.2)$ & $1487(49.7)$ & $337(25.3)$ & $<0.001$ \\
\hline heterosexual & $1573(36.4)$ & $864(28.9)$ & 709 (53.3) & $<0.001$ \\
\hline drug user & $177(4.1)$ & $133(4.4)$ & $44(3.3)$ & 0.097 \\
\hline others/unknown & $749(17.3)$ & $508(17.0)$ & $241(18.1)$ & 0.384 \\
\hline Age (years), median (IQR) & $37(30-45)$ & $38(31-45)$ & $36(29-43)$ & $<0.001$ \\
\hline CD4 cell count (cells $/ \mathrm{mm}^{3}$ ), median (IQR) & $321(132-510)$ & $331(131-552)$ & $304(132-486)$ & 0.006 \\
\hline Viral load $\left(\log _{10}\right.$ copies $/ \mathrm{mL}$ ), median (IQR) & $4.9(4.3-5.4)$ & $4.9(4.3-5.4)$ & $4.9(4.3-5.5)$ & 0.07 \\
\hline Year of diagnosis, median (IQR) & $2010(2007-12)$ & $2009(2007-12)$ & $2011(2008-12)$ & $<0.001$ \\
\hline Year of GRT, median (IQR) & $2010(2007-12)$ & $2010(2007-12)$ & $2011(2008-12)$ & $<0.001$ \\
\hline State of infection, recent, $n(\%)^{b}$ & $403(22.5)$ & $284(22.0)$ & $119(23.7)$ & 0.45 \\
\hline Patients with TDR, $n(\%)$ & $382(8.8)$ & $290(9.7)$ & $92(6.9)$ & 0.003 \\
\hline Patients with NRTI TDR, n (\%) & $152(3.5)$ & $134(4.5)$ & $18(1.4)$ & $<0.001$ \\
\hline Patients with NNRTI TDR, n (\%) & $180(4.2)$ & $124(4.1)$ & $56(4.2)$ & 0.924 \\
\hline Patients with PI TDR, n (\%) & $101(2.3)$ & $75(2.5)$ & $26(1.9)$ & 0.316 \\
\hline Patients in TCs, $n(\%)$ & $954(22.1)$ & $683(22.8)$ & $271(20.4)$ & 0.071 \\
\hline
\end{tabular}

GRT, genotypic resistance test; TDR, transmitted drug resistance; TCs, transmission clusters.

${ }^{a}$ By $\chi^{2}$ test or Fisher's exact test, as appropriate (qualitative variables) and Wilcoxon-Mann-Whitney (quantitative variables).

bData available for 1292 B and 502 non-B subtype-infected individuals.

and $C(219,5.1 \%)$. The remaining individuals were infected with other pure subtypes or CRFs $(535,12.4 \%)$. An increase in non-B HIV-1 subtype infections was found over time $(<2005-14$ : $19.5 \%-38.5 \%, P<0.0001$ ) (Figure $1 \mathrm{a})$. Of note, the proportion of nonB subtypes progressively rose in Italians (<2005-14: 6.5\%-28.8\%, $P<0.0001$ ) (Figure 1b). Regarding risk factors, while individuals infected with B subtype were predominantly MSM (1487, 49.7\%), non-B subtype-infected individuals mainly reported heterosexual contact (709, 53.3\%) (Table 1). However, looking at the dynamics of risk factors in the non-B-infected population, a significant increase was observed in the MSM over time (<2005-14: $20.0 \%-36.0 \%$, $P<0.0001)$. This phenomenon is strictly related to the recent increasing proportion of Italian MSM in this non-B population $(<2005-14$ : $50.0 \%-80.4 \%, P=0.047)$. Interestingly, the prevalence of MSM also increased over time in the 403 recent infections $(<2005-14$ : 45.5\%-78.6\%, $P<0.0001)$.

\section{Prevalence and temporal trend of transmitted drug resistance}

The overall prevalence of TDR in the period 2000-14 was 8.8\% $(N=382)$. The majority of individuals carried a single mutation $(N=266,69.6 \%)$. TDR was higher in B subtype-infected individuals than in those infected with non-B subtypes (9.7\% versus $6.9 \%$, $P=0.003)$. By analysing individuals carrying TDR and with an available state of infection, 38 (26.2\%) were in recent infections.
Of these, 24 (63.1\%) were MSM and 12 (31.6\%) were heterosexual transmissions.

Analysing the TDR temporal trend, in B subtype-infected individuals resistance to any drug class decreased over time with a slight trend towards significance (<2005-14: 13.4\%-8.1\%, $P=0.156)$, with a significant decrease in DR to PIs (<2005-14: 5.0\%-1.6\%, $P=0.001$ ) (Figure 2a). In contrast, among the 1331 non-B subtypeinfected individuals, TDR to any drug class significantly increased over time ( $<2005-14: 2 \%-7.1 \%, P=0.018$ ) (Figure $2 b)$.

\section{Transmission clusters and their role in TDR}

Overall, we identified 467 TCs, involving 1207 of the 4323 newly diagnosed individuals analysed (27.9\%). Among B subtypeinfected subjects, 259 pairs and 82 TCs with $\geq 3$ individuals were identified, while 86 pairs and 40 TCs ( $\geq 3$ individuals) were detected in non-B subtype-infected individuals.

Even if the prevalence of sequences grouping in TCs was only $27.9 \%$, an increasing proportion of individuals in B subtype TCs $(<2005-14: 12.9 \%-33.5 \%, P=0.001)$ and non-B subtype TCs (<2005-14: 18.4\%-41.9\%, $P=0.006)$ was observed over time (data not shown).

With regard to characteristics of individuals involved in TCS, women and heterosexuals were more frequently found in non-B subtype TCs than in B subtype TCs (22.4\% versus $12.1 \%$, $P<0.0001$, and $42.1 \%$ versus $25.4 \%, P<0.0001$, respectively). 

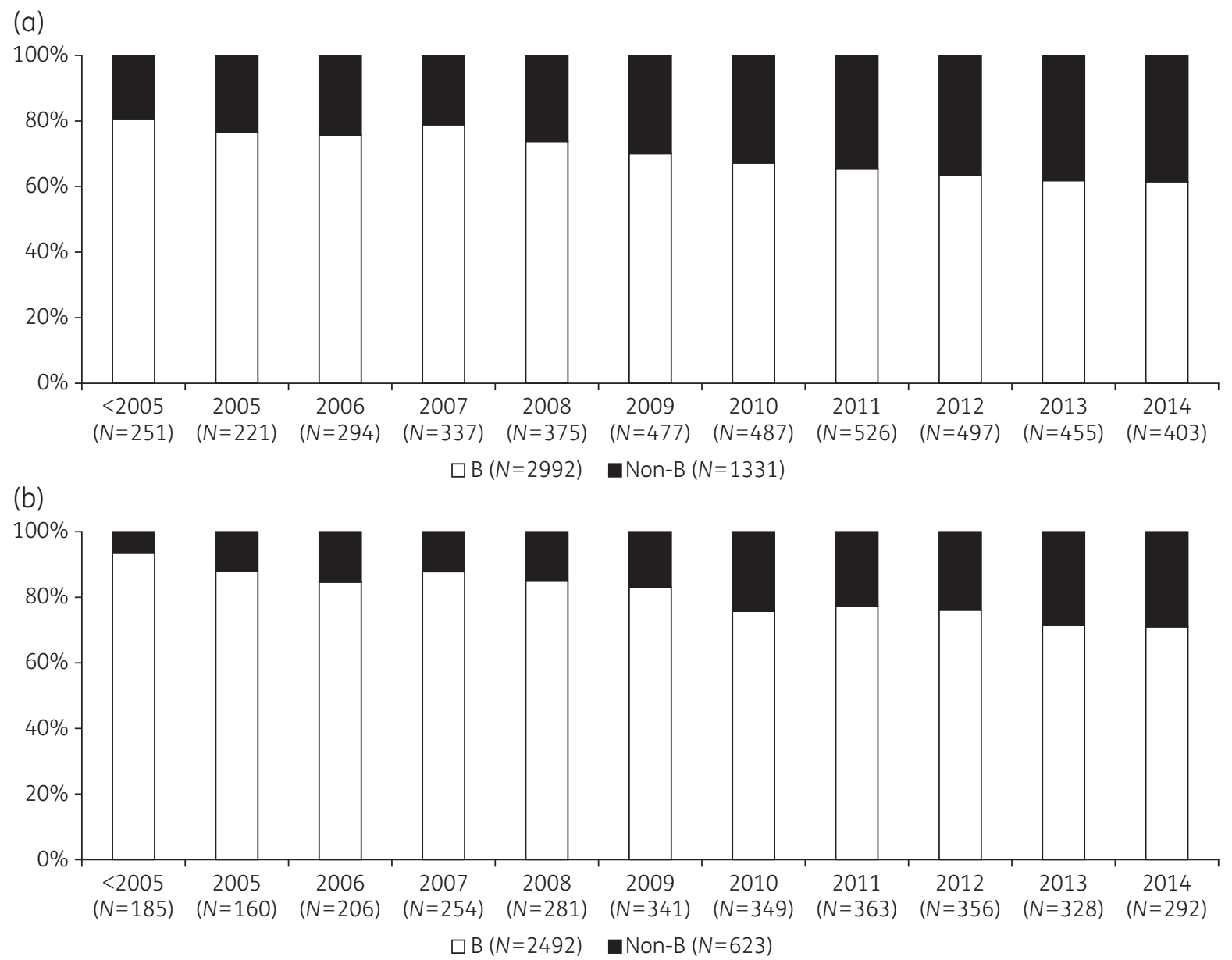

Figure 1. Evaluation of B and non-B subtype prevalence in HIV-1-infected individuals over time in the overall population (a) and in Italians (b). $P$ values (calculated by time series analysis for linear trend over time) were both significant $(P<0.0001)$.

Moreover, individuals in non-B subtype TCs were diagnosed in more recent years compared with those involved in B subtype TCs [2011 (2009-13) versus 2010 (2008-12), P<0.0001]. Considering the state of infection, $58.5 \%$ of recent infections in TCS involved MSM, while only $31.9 \%$ involved heterosexuals $(P=0.11)$.

Looking at resistance, $13.3 \%$ of TCs ( $N=62$; B subtype: $N=44$, non-B subtypes: $N=18$ ) involved at least one subject with TDR (112 individuals, $29.3 \%$ of the entire TDR population) (Figures S1 and S2, available as Supplementary data at JAC Online). Of these $62 \mathrm{TCS}, 48$ (B subtype: $N=35$; non-B subtypes: $N=13$ ) were pairs, and 14 (B subtype: $N=9$; non-B subtypes: $N=5$ ) were TCs with $\geq 3$ individuals. Moreover, among these 62 TCs, 24 (38.7\%) included a single patient with TDR, while $32(51.6 \%)$ were entirely composed of individuals carrying resistance (Table S1).

By analysing the rate of TDR clustering over time, the presence of TDR within TCs dramatically increased over the years, reaching $35.5 \%$ of the overall TDR in $2014(<2005-14: 14.3 \%-35.5 \%$, $P=0.005)$ (data not shown).

Considering the characteristics of the 190 subjects involved in the 62 TDR TCs, 86.3\% ( $N=165)$ were male, $79.5 \%(N=151)$ were Italians and $49.5 \%(N=94)$ were MSM (Table S1). By comparing the characteristics between $B$ and non-B subtype TDR TCS, non-B subtype-infected subjects were diagnosed in more recent years [2012 (2010-13) versus 2009 (2008-12), $P<0.0001]$, and more frequently involved in large TCs [median 7 (IQR 6-19) versus 4 (3-4), $P=0.047$ ] compared with individuals infected with a B subtype. No statistically significant differences were found in the time of infection (recent or long-standing), nationalities or risk factors.

\section{Prevalence of TDR mutations in the overall population and in the TCS}

The prevalence of TDR mutations in the overall population and in the TCs according to drug classes and subtypes is shown in Figure 3.

Analysing resistance in HIV-1 B subtype-infected individuals, $4.5 \%$ of them (134/2992) harboured mutations associated with resistance to NRTIs; of note, $1.4 \%$ of these individuals (41/2992) were involved in TCS. The NRTI mutation T215rev had the highest prevalence in the overall B subtype-infected population (3.1\%: individuals involved in TCs, $0.9 \%$; individuals not included in TCS, $2.2 \%$ ), followed by M41L (1.6\%: $0.6 \%$ and $1 \%$ ) and L210W (1.0\%: $0.3 \%$ and $0.7 \%)$. Interestingly, in non-B subtype-infected individuals NRTI mutations accounted only for $1.4 \%$ of the overall population; none of these mutations were found in TCs. 

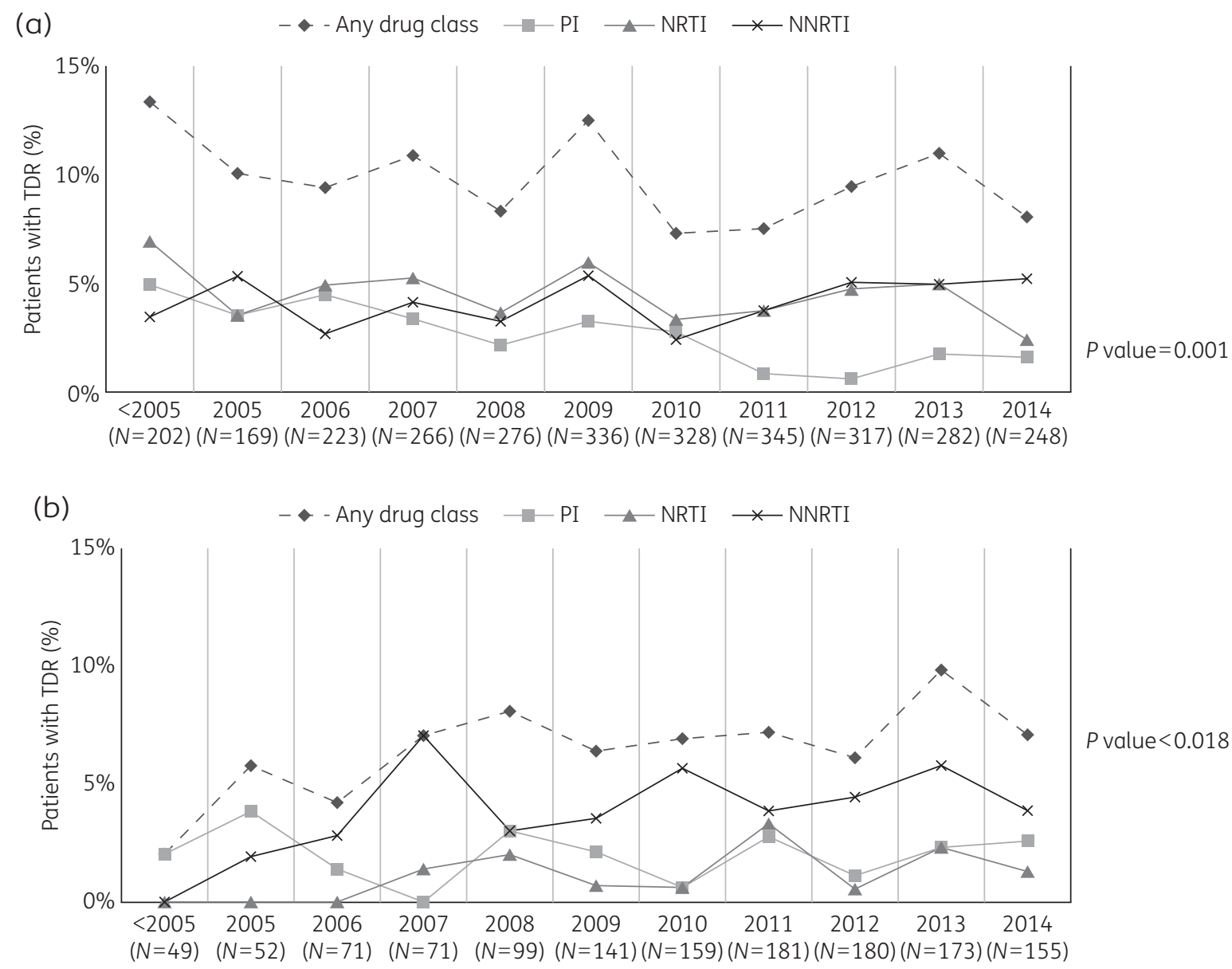

Figure 2. Prevalence of transmitted drug resistance (TDR) in Italian HIV-1-infected individuals, diagnosed in the period 2000-14, according to calendar year and drug classes. (a) Prevalence of TDR over time in individuals infected with HIV-1 B subtype. (b) Prevalence of TDR over time in individuals infected with HIV-1 non-B subtypes. The $P$ value was calculated by time-series analysis for linear trend over time (only significant values are shown).

Concerning NNRTI resistance, $4.1 \%(124 / 2992)$ and $4.2 \%$ (56/1331) of the B and non-B subtype infected individuals carried at least one mutation to this class, respectively. Of note, $1.0 \%$ of $B(31 / 2992)$ and $1.9 \%$ of non-B (25/1331) subtype-infected individuals were involved in TCs.

In both $B$ and non-B subtype groups, the most prevalent NNRTI mutations (either in individuals included or in those non-included in TCs) were K103N (B subtype: 2.1\%; non-B subtype: $1.7 \%$ ) and E138G/K (0.7\%; 1.7\%), followed by K101E $(0.7 \% ; 0.8 \%)$.

In the overall $B$ and non-B subtype groups, mutations associated with resistance to PI were observed with a prevalence $2.5 \%$ (individuals involved in TCs: $0.6 \%$; individuals not included in TCs: $1.9 \%)$ and $2.0 \%(0.7 \% ; 1.3 \%)$; Q58E was the most prevalent PI mutation found both in the overall B and non-B groups.

\section{Factors associated with TDR}

Univariate and multivariate logistic regression models were performed to identify potential predictors of TDR (Figure 4).

The results showed that in our population, being part of TCs was not a predictor of TDR [adjusted odds ratio, AOR (95\% CI): 0.92 (0.71-1.18); $P=0.495]$. Whereas there was a negative association between the presence of TDR and individuals infected with a CRF02_AG recombinant form compared with those infected with a B subtype [AOR ( $95 \%$ CI): $0.45(0.26-0.80) ; P=0.006]$. These results were also confirmed by using the type of infection as a confounder ( $N=1794)$, (Figure 4b).

\section{Discussion}

We have here described molecular and epidemiological characteristics of $4323 \mathrm{HIV}-1$ drug-naive infected individuals attending three different counselling and testing centres in north and central Italy in the period 2000-14 to gain further insights into the time trends of subtype distribution, TDR and TCs.

The first finding concerns the epidemiological changes occurring in Italy over the last decade. In particular, our study, which covers a period of 14 years, observed a higher prevalence of non-B subtypes in newly diagnosed individuals, and, mainly, in subjects originating from Italy. This is in line with the important increase of non-B subtypes and CRFs reported in several European countries ${ }^{15,38-43}$ during the last decade. This increase in non-B subtypes occurred in conjunction with associated epidemiological changes, such as waves of migration from low-income to middle-income areas, ${ }^{18,19}$ 
(a)

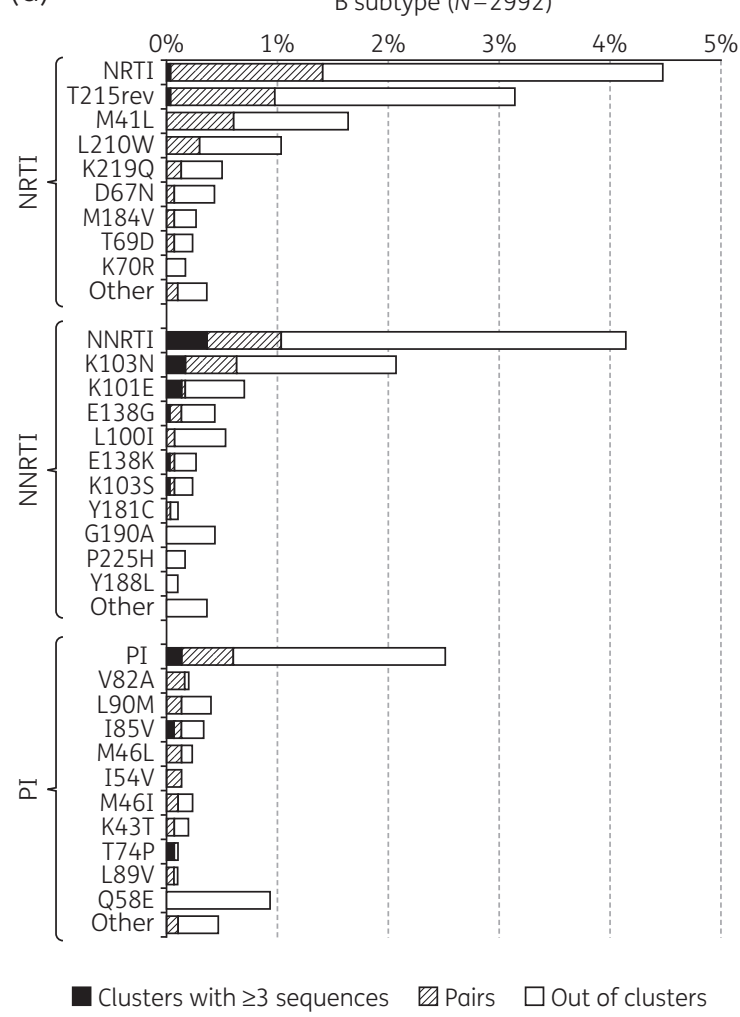

(b)

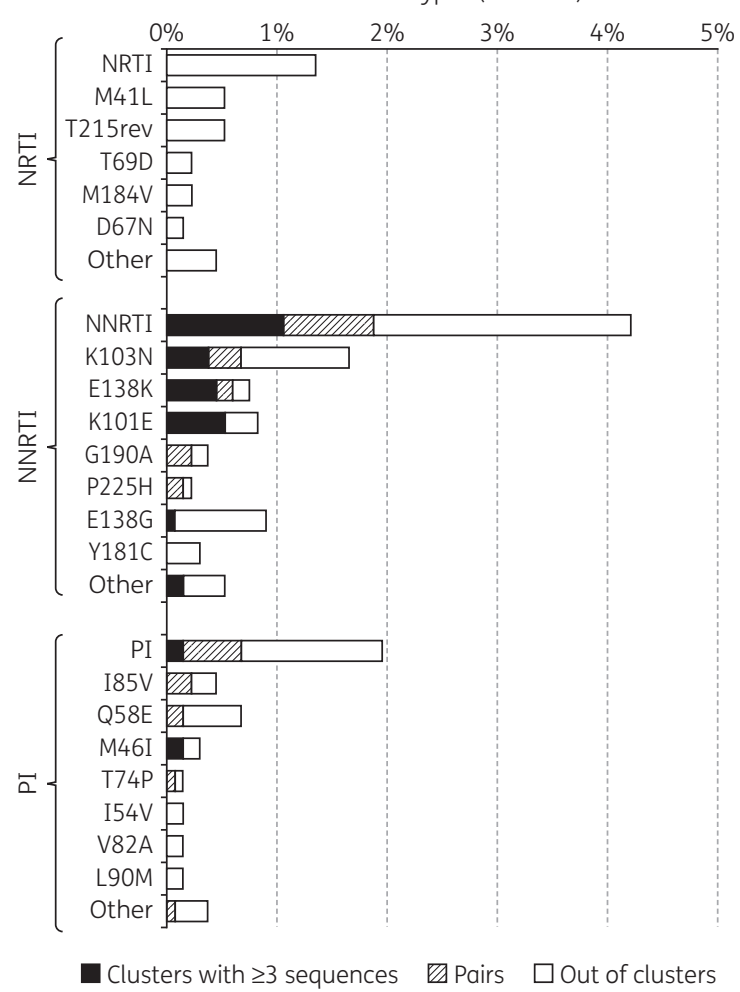

Figure 3. Prevalence of drug resistance mutations in HIV-1 B (a) and non-B subtypes (b) according to drug classes and presence or not in transmission clusters (TCs). Each bar is divided according to: (i) the presence of drug resistance mutations in clusters with $\geq 3$ sequences (black bar); (ii) the presence of drug resistance mutations in pairs (hatched bar); (iii) the presence of drug resistance mutations out of clusters (white bar). 'Other' includes all the resistance mutations with an overall prevalence $<0.2 \%$.

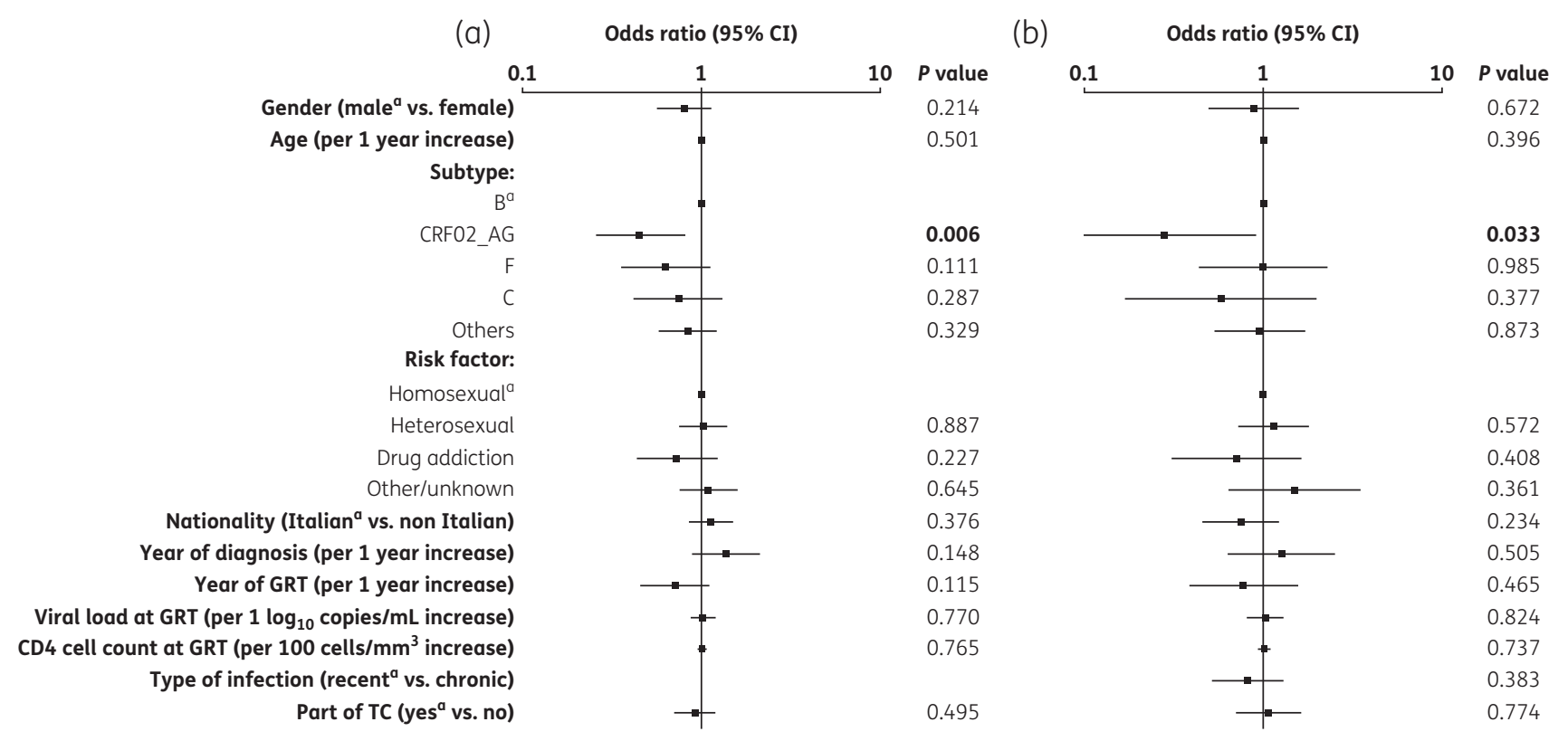

Figure 4. (a) Multivariable logistic regression model performed on the overall population analysed. The model was adjusted for gender, age, subtype, risk factor, nationality, year of diagnosis, year of genotypic resistance testing (GRT), viral load at GRT (log 10 copies/mL), CD4 cell count at GRT (cells $/ \mathrm{mm}^{3}$ ), and being part of a transmission cluster (TC). (b) Multivariable logistic regression model performed on a subset of individuals ( $\mathrm{N}=1794$, with similar characteristics of the overall population) for whom type of infection (recent or chronic) was known. The model was adjusted for the same variables included in the model shown in (a) and the type of infection. 
and an increase in HIV transmission among MSM. ${ }^{20,44-49}$ In line with this phenomenon, our study also shows that the non-B epidemic is characterized by a shift in the prevalent modality of infection and nationality, and also in the setting of recent infection. Based on these findings, we analysed the dynamics of TDR according to these temporal epidemiological changes.

The overall TDR prevalence was $8.8 \%$, consistent with other European studies, ${ }^{11-13,50,51}$ but lower than that observed in several US settings. ${ }^{8,9}$ However, an alarming temporal TDR increase was observed in subjects infected with non-B subtype HIV-1 (<2005-14: 2.0\%-7.1\%, $P=0.018$ ), different from that observed in HIV-1 B-infected individuals. This increasing trend of TDR in nonB subtypes observed in our population coincided with an increase in recent infections characterized by a homosexual transmission route. This finding was also reported by a recent paper published by our group, ${ }^{20}$ highlighting circulation of TDR TCs characterized by non-B MSM. Other European studies confirmed the strict relationship between the increasing trend of TDR and the MSM route, in non-B infections, ${ }^{49,51}$ but also in subtype $B$ infections. ${ }^{11}$ These data highlight the importance of studying and monitoring local epidemics and suggests TDR prevalence may vary within subtypes and risk factors.

By assessing TDR prevalence according to drug class, we found that the increasing trend in non-B TDR is mainly linked to the detection of NNRTI DR, as also suggested by the high prevalence of K103N and K101E in the overall and TC populations. The same linear increase was observed in the SPREAD study that included data up to $2007,{ }^{52}$ in which the authors suggested a potential association with the frequent use of NNRTI in the first-line regimens. Indeed, from 2009 onwards, the most commonly prescribed regimen was the potent combination tenofovir + emtricitabine + efavirenz, and up until 2012, no other available regimens had proven superior to this one with respect to the virological response.

The second aim of this study was to evaluate the contribution of TCs in the spread of the HIV epidemic and TDR. In this study, the rate of sequences segregating in clusters is $28.0 \%$, similar to that reported in France, ${ }^{40}$ the Basque country ${ }^{53}$ and the UK, ${ }^{54}$ and quite low with respect to that reported in the Nordic European countries $^{41}$ and in the Swiss cohort. ${ }^{54}$

The presence of TDR within TCs accounted for $29.3 \%$ of the entire TDR population. The contribution of TCs to TDR observed was superimposable onto those reported by other European and US studies, ${ }^{7,11}$ especially when a more stringent criterion of genetic distance for TC detection was applied. ${ }^{11}$ Most importantly, our study indicates that the contribution of TCs to the spread of DR has increased in the last few years, with a peak in 2014, suggesting that a public health policy targeting early diagnoses is needed. Since multivariate logistic regression models did not identify factors associated with TDR, with the exception of a negative role of CRF02_AG, we were unable to identify positive predictors of DR, a result similar to that observed in other studies performed in Europe. ${ }^{11,14}$ In line with other European cohorts, our TDR TCs frequently involved individuals with different subtypes and nationalities. $^{11,20}$ This implies that migration plays an important role in the local spread of B and non-B subtypes, but also of TDR, supporting an extensive intermixing of the HIV-1 epidemic between locals and immigrants.

Moreover, as reported above, the spread of non-B subtypes in the local epidemic and in TCs was an increasing phenomenon, mainly related to the MSM route that often involves Italians. The contribution of MSM in TCs was also evident when recent infections were considered. In particular, $30.3 \%$ of MSM in our TC population was recently infected and this prevalence increased to $44.4 \%$ when non-B TCs were considered. The high propensity of MSM for clustering was previously reported by Ragonnet-Cronin et al., ${ }^{54}$ who found a predominant MSM contribution in TCs for both the UK HIV Drug Resistance Database and the Swiss HIV Cohort Study.

Regarding TDR in TCS, NRTIs, and in particular the T215 revertant mutations, were predominant in B subtype TCs, confirming the frequent transmission of viruses containing revertants at position 215 in Europe. ${ }^{11,33}$ In contrast, we did not find any resistance mutations associated with thymidine analogues (such as T215rev, M41L and L210W) in non-B TCs. This finding may be explained by the more recent date of diagnosis found in non-B versus $B$ subtypes, occurring when the use of thymidine analogues was no longer recommended.

In non-B TCs, the predominant role in the spread of TDR was played by NNRTI mutations (in particular K103N, E138K and K101E). In fact, one of the most important TCs carried K103N (shown in Figure S2). This cluster involved both heterosexual and homosexual males originating from Italy. The latest diagnosis date was 2014, suggesting that in our local epidemic this mutation was involved in a recent spread.

Thus, continuous monitoring of the role of NNRTI mutations in TCs is required to establish their impact on current HIV epidemics.

As with any other observational study, our data may have some limitations. First, our study population might not be representative of the overall Italian population. In particular, looking at the HIV/AIDS surveillance reports published by ECDC and the WHO Regional Office for Europe (WHO/Europe), ${ }^{22} 37023$ new diagnoses of HIV-1 were reported in Italy from 2005 to 2015. Thus, the 4323 HIV-1 diagnoses reported in our study in the same time period represent $11.7 \%$ of the overall HIV-1-infected Italian population. Focusing attention on the new diagnoses found in north and central Italy $(N=22798)$, this coverage increased to $19.0 \%$ of the overall HIV-1-infected individuals. ${ }^{55}$ However, the increasing prevalence of non-B subtypes and MSM found in our population over time (also in the setting of recent infections) is confirmed by the high prevalence of immigrants and MSM reported in 2015, ${ }^{55}$ thus suggesting that our samples broadly matched the characteristics of the whole HIV-1-infected Italian population. The increasing trend of non-B and MSM was also reported by several European studies, ${ }^{41,44,48,49}$ suggesting a good reproducibility of our results with respect to large European contexts, and supporting the role of migration in the epidemiological changes described above.

Second, this study did not evaluate TDR to other classes of antiretroviral drugs, such as integrase inhibitors. As the use of these agents increases and is recommended by national and international guidelines, 5,56,57 surveillance for TDR must be included. Finally, the potential association between demographic data and TDR could not be evaluated in depth because some information was not available.

In conclusion, this study proves that complementing information on individuals' demographics, modality of transmission, viral and clinical parameters, with results obtained through phylogenetic analysis, creates an added value in the understanding of local HIV epidemics. In particular, with this study, we were able to show that individuals infected with B subtype viruses and individuals 
infected with non-B subtypes represent significantly different populations, with different routes of transmission and different trends of TDR. This study also suggests that HIV-1 non-B subtypes in Italy may have a primary role in the spread of drug-resistant strains, which have to date mainly been associated with the B subtype. Indeed, TDR and complex TCs containing drug-resistant strains, to date observed mainly in the context of B subtype infections, dramatically increased in HIV-1 non-B-infected individuals.

Overall, this deeper analysis can contribute to the design of well-targeted prevention and control programmes, and to reinforce the current recommendation for both baseline resistance testing to guide treatment decisions and early treatment in order to prevent transmission of HIV drug resistance. ${ }^{5,6}$

\section{Acknowledgements}

We gratefully thank A. Biddittu, M. Bruni, A. Giannetti, M. Romani and A. Pacifici for data management.

The manuscript was presented in part at the International HIV Drug Resistance Workshop, 20-21 February 2016, Boston, Massachusetts USA (Abstract 74).

\section{SENDIH (Studio Epidemiologico Nuove Diagnosi Infezione da HIV) Study Group Members}

E. Girardi, M. R. Capobianchi, C. F. Perno, N. Orchi, A. Navarra, A. Palummieri, I. Abbate, A. Ammassari, R. D’Arrigo, G. De Carli, L. Fabeni, F. Forbici, F. M. Fusco, C. Gori, S. Grisetti, A. Mariano, E. Nicastri, G. Nurra, C. Pinnetti, S. Pittalis, V. Puro, A. Sampaolesi, M. R. Sciarrone, P. Scognamiglio, M. Selleri, C. Sias, M. Zaccarelli, (National Institute for Infective Diseases, 'L. Spallanzani', Rome); A. Di Carlo, M. Giuliani (Division of Dermatological Infectious Diseases, STI/HIV Unit, San Gallicano Institute, Rome); V. Vullo, M. Falciano (CRAIDS Sapienza University, Rome); A. Pennica (UO AIDS S. Andrea Hospital, Rome), F. Errigo (UO AIDS ASL RMB S. Pertini Hospital, Rome); P. Gattari, L. Spizzichino (UO AIDS ASL RME, Rome); S. Schito (UO AIDS ASL RMD GB Grassi Hospital, Rome); M. Andreoni, L. Sarmati, A. R. Buonomini, C. Cerva (Clinical Infectious Diseases Unit, Tor Vergata University, Roma), C. Mastroianni, M. Lichtner (CRAIDS Hospital, Latina); V. S. Mercurio, E. Anzalone (CRAIDS Hospital, Frosinone); A. Pitorri (CRAIDS Hospital, Rieti); A. Caterini, S. Aviani Barbacci (CRAIDS Hospital, Viterbo).

\section{Funding}

This work was financially supported by the European Commission Framework 7 Programme (CHAIN, the Collaborative HIV and Anti-HIV Drug Resistance Network, Integrated Project No. 223131), the Italian Ministry of Health (Progetto Ricerca Corrente 2016, line no. 2, project no. 2, sub-project no. 2d), the Italian Ministry of Education, University and Research (MIUR) (Bandiera InterOmics Protocollo PB051, PRIN 2012, Protocollo 2012L783TW) and an unrestricted grant from AVIRALIA Foundation.

\section{Transparency declarations}

None to declare.

\section{Supplementary data}

Table S1 and Figures S1 and S2 appear as Supplementary data at JAC Online.

\section{References}

1 Little SJ, Holte S, Routy JP et al. Antiretroviral-drug resistance among patients recently infected with HIV. N Engl J Med 2002; 347: 385-94.

2 Violin M, Cozzi-Lepri A, Velleca R et al. Risk of failure in patients with 215 HIV-1 revertants starting their first thymidine analog-containing highly active antiretroviral therapy. AIDS 2004; 18: 227-35.

3 Kuritzkes DR, Lalama CM, Ribaudo HJ et al. Preexisting resistance to nonnucleoside reverse-transcriptase inhibitors predicts virologic failure of an efavirenz-based regimen in treatment-naive HIV-1-infected subjects. J Infect Dis 2008; 197: 867-70.

4 Wittkop L, Günthard HF, de Wolf F et al. Effect of transmitted drug resistance on virological and immunological response to initial combination antiretroviral therapy for HIV (EuroCoord-CHAIN joint project): a European multicohort study. Lancet Infect Dis 2011; 11: 363-71.

5 European AIDS Clinical Society. EACS Guidelines Version 8.0. 2016. http:// www.eacsociety.org/guidelines/eacs-guidelines/eacs-guidelines.html.

6 Günthard HF, Aberg JA, Eron JJ et al. Antiretroviral treatment of adult HIV infection: 2014 recommendations of the International Antiviral Society-USA Panel. JAMA 2014; 312: 410-25.

7 Panichsillapakit T, Smith DM, Wertheim JO et al. Prevalence of transmitted HIV drug resistance among recently infected persons in San Diego, CA 19962013. J Acquir Immune Defic Syndr 2016; 71: 228-36.

8 Youmans E, Tripathi A, Albrecht $\mathrm{H}$ et al. Transmitted antiretroviral drug resistance in individuals with newly diagnosed HIV infection: South Carolina 2005-2009. South Med J 2011; 104: 95-101.

9 Sey K, Ma Y, Lan YC et al. Prevalence and circulation patterns of variant, atypical and resistant HIV in Los Angeles County (2007-2009). J Med Virol 2014; 86: 1639-47.

10 Tostevin A, White E, Dunn D et al. Recent trends and patterns in HIV-1 transmitted drug resistance in the United Kingdom. HIV Med 2016; 18: 204-13.

11 Pineda-Peña AC, Schrooten $Y$, Vinken $L$ et al. Trends and predictors of transmitted drug resistance (TDR) and clusters with TDR in a local Belgian HIV-1 epidemic. PLoS One 2014; 9: e101738.

12 Vega Y, Delgado E, Fernández-García A et al. Epidemiological surveillance of HIV-1 transmitted drug resistance in Spain in 2004-2012: relevance of transmission clusters in the propagation of resistance mutations. PLoS One 2015; 10: e0125699.

13 Frange P, Assoumou L, Descamps D et al. HIV-1 subtype B-infected MSM may have driven the spread of transmitted resistant strains in France in 2007-12: impact on susceptibility to first-line strategies. I Antimicrob Chemother 2015; 70: 2084-9.

14 Yebra G, Delgado R, Pulido F et al. Different trends of transmitted HIV-1 drug resistance in Madrid, Spain, among risk groups in the last decade. Arch Virol 2014; 159: 1079-87.

15 Giuliani M, Santoro MM, Lo Presti A et al. Circulation of HIV-1 CRF02_AG among MSM population in central Italy: a molecular epidemiology-based study. Biomed Res Int 2013; 2013: 810617.

16 Chaix ML, Seng R, Frange P et al. Increasing HIV-1 non-B subtype primary infections in patients in France and effect of HIV subtypes on virological and immunological responses to combined antiretroviral therapy. Clin Infect Dis 2013; 56: 880-7. 
17 Yebra G, de Mulder M, Martín L et al. Most HIV type 1 non-B infections in the Spanish cohort of antiretroviral treatment-naive HIV-infected patients (CoRIS) are due to recombinant viruses. J Clin Microbiol 2012; 50: 407-13.

18 Gifford RJ, de Oliveira T, Rambaut A et al. Phylogenetic surveillance of viral genetic diversity and the evolving molecular epidemiology of human immunodeficiency virus type 1. J Virol 2007; 81: 13050-6.

19 Lospitao E, Alvarez A, Soriano V et al. HIV-1 subtypes in Spain: a retrospective analysis from 1995 to 2003. HIV Med 2005; 6: 313-20.

20 Fabeni L, Alteri C, Orchi $\mathrm{N}$ et al. Recent transmission clustering of HIV-1 C and CRF17_BF strains characterized by NNRTI-related mutations among newly diagnosed men in central Italy. PLoS One 2015; 10: e0135325.

21 Fox J, Castro H, Kaye S et al. Epidemiology of non-B clade forms of HIV-1 in men who have sex with men in the UK. AIDS 2010; 24: 2397-401.

22 ECDC: Surveillance Report. HIV/AIDS Surveillance in Europe. 2016. http:// ecdc.europa.eu/en/healthtopics/aids/Pages/surveillance-reports.aspx.

23 Hué S, Brown AE, Ragonnet-Cronin M et al. Phylogenetic analyses reveal HIV-1 infections between men misclassified as heterosexual transmissions. AIDS 2014; 28: 1967-75.

24 Zeh C, Inzaule SC, Ondoa P et al. Molecular epidemiology and transmission dynamics of recent and long-term HIV-1 infections in rural Western Kenya. PLoS One 2016; 11: e0147436.

25 Kassaye SG, Grossman Z, Balamane M et al. Transmitted HIV drug resistance is high and longstanding in metropolitan Washington, DC. Clin Infect Dis 2016; 63: 836-43.

26 Suligoi B, Buttò S, Galli C et al. Detection of recent HIV infections in African individuals infected by HIV-1 non-B subtypes using HIV antibody avidity. J Clin Virol 2008; 41: 288-92.

27 Selleri M, Orchi N, Zaniratti MS et al. Effective highly active antiretroviral therapy in patients with primary HIV-1 infection prevents the evolution of the avidity of HIV-1-specific antibodies. J Acquir Immune Defic Syndr 2007; 46: 145-50.

28 Armenia D, Di Carlo D, Maffongelli G et al. Virological response and resistance profile in HIV-1-infected patients starting darunavir-containing regimens. HIV Med 2017; 18: 21-32.

29 Saitou N, Nei M. The neighbor-joining method: a new method for reconstructing phylogenetic trees. Mol Biol Evol 1987; 4: 406-25.

30 Kimura M. A simple method for estimating evolutionary rates of base substitutions through comparative studies of nucleotide sequences. J Mol Evol 1980; 16: 111-20.

31 Bennett DE, Camacho RJ, Otelea D et al. Drug resistance mutations for surveillance of transmitted HIV-1 drug-resistance: 2009 update. PLoS One 2009; 4: e4724.

32 Wensing AM, Calvez V, Günthard HF et al. 2017 Update of the drug resistance mutations in HIV-1. Top Antivir Med 2017; 24: 132-3.

33 Chalmet K, Staelens D, Blot S et al. Epidemiological study of phylogenetic transmission clusters in a local HIV-1 epidemic reveals distinct differences between subtype B and non-B infections. BMC Infect Dis 2010; 10: 262.

34 Tavaré S. Some probabilistic and statistical problems in the analysis of DNA sequences. Lectures Math Life Sci 1986; 17: 57-86.

35 Ragonnet-Cronin M, Hodcroft E, Hué S et al. Automated analysis of phylogenetic clusters. BMC Bioinformatics 2013; 14: 317.

36 Huelsenbeck JP, Ronquist F. MRBAYES: Bayesian inference of phylogenetic trees. Bioinformatics 2001; 17: 754-5.

37 Ronquist F, Huelsenbeck JP. MrBayes 3: Bayesian phylogenetic inference under mixed models. Bioinformatics 2003; 19: 1572-4.

38 Lai A, Riva C, Marconi A et al. Changing patterns in HIV-1 non-B clade prevalence and diversity in Italy over three decades. HIV Med 2010; 11: 593-602.
39 Lai A, Bozzi G, Franzetti M et al. HIV-1 A1 subtype epidemic in Italy originated from Africa and Eastern Europe and shows a high frequency of transmission chains involving intravenous drug users. PLoS One 2016; 11: e0146097.

40 Chaillon A, Essat A, Frange $P$ et al. Spatiotemporal dynamics of HIV-1 transmission in France (1999-2014) and impact of targeted prevention strategies. Retrovirology 2017; 14: 15.

41 Esbjörnsson J, Mild M, Audelin A et al. HIV-1 transmission between MSM and heterosexuals, and increasing proportions of circulating recombinant forms in the Nordic Countries. Virus Evol 2016; 2: vew010.

42 Niculescu I, Paraschiv S, Paraskevis D et al. Recent HIV-1 outbreak among intravenous drug users in Romania: evidence for cocirculation of CRF14_BG and subtype F1 strains. AIDS Res Hum Retroviruses 2015; 31: 488-95.

43 Patiño Galindo JA, Torres-Puente M, Gimeno C et al. Expansion of the CRF19_cpx variant in Spain. J Clin Virol 2015; 69: 146-9.

44 Semaille C, Cazein F, Lot F et al. Recently acquired HIV infection in men who have sex with men (MSM) in France, 2003-2008. Euro Surveill 2009; 14: pii $=19425$.

45 Notiziario dell'Istituto Superiore di Sanità, vol. 26, numero 9, supp. 1. http://www.salute.gov.it/imgs/C_17_pubblicazioni_2065_allegato.pdf.

46 Brenner BG, Ibanescu RI, Hardy I et al. Large cluster outbreaks sustain the HIV epidemic among MSM in Quebec. AIDS 2017; 31: 707-17.

47 Drescher SM, von Wyl V, Yang WL et al. Treatment-naive individuals are the major source of transmitted HIV-1 drug resistance in men who have sex with men in the Swiss HIV Cohort Study. Clin Infect Dis 2014; 58: 285-94.

48 Patiño-Galindo JÁ, Torres-Puente M, Bracho MA et al. Identification of a large, fast-expanding HIV-1 subtype B transmission cluster among MSM in Valencia, Spain. PLoS One 2017; 12: e0171062.

49 Parczewski M, Leszczyszyn-Pynka M, Witak-Jędra M et al. Expanding HIV1 subtype $B$ transmission networks among men who have sex with men in Poland. PLoS One 2017; 12: e0172473.

50 Descamps D, Assoumou L, Chaix ML et al. National sentinel surveillance of transmitted drug resistance in antiretroviral-naive chronically HIV-infected patients in France over a decade: 2001-2011. J Antimicrob Chemother 2013; 68: 2626-31.

51 Lunar MM, Židovec Lepej S, Abecasis AB et al. Short communication: prevalence of HIV type 1 transmitted drug resistance in Slovenia: 2005-2010. AIDS Res Hum Retroviruses 2013; 29: 343-9.

52 Frentz D, Van de Vijver DA, Abecasis AB et al. The epidemiology of HIV-1 transmitted drug resistance: increase in transmitted resistance to nonnucleoside reverse transcriptase inhibitors among newly diagnosed HIV-1 infections in Europe. BMC Infect Dis 2014; 14: 407.

53 Patiño-Galindo JA, Thomson MM, Pérez-Álvarez L et al. Transmission dynamics of HIV-1 subtype B in the Basque Country, Spain. Infect Genet Evol 2016; 40: 91-7.

54 Ragonnet-Cronin ML, Shilaih M, Günthard HF et al. A direct comparison of two densely sampled HIV epidemics: the UK and Switzerland. Sci Rep 2016; 6 : 32251.

55 Notiziario dell'Istituto Superiore di Sanità, vol. 29, numero 9, supp. 1. http://www.iss.it/binary/publ/cont/COA_ONLINE_2016.pdf.

56 Linee Guida Italiane sull'utilizzo dei farmaci antiretrovirali e sulla gestione diagnostico-clinica delle persone con infezione da HIV-1. Società italiana di malattie infettive e tropicali e Ministero della Salute. http://www.salute.gov.it/ portale/temi/p2_6.jsp?lingua=italiano\&id=187\&area=aids\&menu=vuoto.

57 Panel on Antiretroviral Guidelines for Adults and Adolescents. Guidelines for the Use of Antiretroviral Agents in HIV-1-Infected Adults and Adolescents. Department of Health and Human Services. http://www.aidsinfo.nih.gov/ ContentFiles/AdultandAdolescentGL.pdf. 